\title{
Blockchain Application in Manufacturing Industry - Bibliometric and Systemic Analysis
}

\author{
Gabriel Lodi ${ }^{1}$, Jaqueline Sebastiany Iaksch and Milton Borsato \\ Universidade Tecnológica Federal do Paraná (UTFPR), Campus Curitiba, Brazil \\ Graduate School of Mechanical Engineering and Materials (PPGEM-CT)
}

\begin{abstract}
Blockchain technology provides secure, reliable and transparent solutions to different challenges arising from the growing need for connectivity presented nowadays. Recent developments in industrial processes sets fertile ground for the application of this technology. Aiming to understand the state of the art of blockchain technology application in the manufacturing industry, this study presents an investigation of relevant publications on the subject, including also bibliometric and systemic analyzes. 30 articles of major relevance were selected from a portfolio of 1432 publications, presented between 2015 and 2019. Thus, it was possible to identify research trends and opportunities.
\end{abstract}

Keywords. Blockchain, Industry, Manufacturing, Supply Chain, Smart Contracts

\section{Introduction}

Given the development and advent of increasingly disruptive new technologies, the manufacturing industry is becoming even more connected and automated. Different names and classifications had been given to this recent revolution, such as industry 4.0, cyber-physical manufacturing, intelligent manufacturing and the industrial internet of things [1]. This movement generates competitiveness, efficiency, and a diversity of intelligent solutions. Therefore, needing to process high amounts of data from multiple sources and managing different interfaces from distinct origins, features such as safety, reliability, and traceability become paramount [2].

One possible answer to these different demands is the blockchain technology. Founded in 2008 as a technical solution for the implementation of bitcoin cryptocurrency [3], blockchain presents itself as a distributed, decentralized and inviolable database, while also ensuring the anonymity of the members and complete transactions traceability [4]. The blockchain gather the history of all transactions into a cryptographic data structure grouped into blocks of information. Each block is connected through cryptographic hash functions to the immediate previous block. This mechanism prevents possible tampering attempts into the ledger history, since the minor adulteration would represent different results from the hash functions, breaking the connection of blocks [5]. Blockchain miners are presented as blockchain nodes responsible for receiving the latest transactions, gathering them into new blocks and for spreading them back to the

\footnotetext{
${ }^{1}$ Corresponding Author. Email: gabrielodi@gmail.com
} 
blockchain network [6]. Thereby, not only the storage of the blockchain may be decentralized but also the processing of new events.

The evolution of blockchain into implementations other than bitcoin has enabled the development of the smart contracts solutions, which is a key structure for the feasibility of applications in supply chain environments, process automation, and transaction conditions verifications without the need for human intervention [7].

Influenced by this scenario, this work presents a structured literature review that aims to identify the state of the art and related research opportunities on blockchain technology application into the manufacturing industry. Similar works have been done focusing on blockchain literature review for specific fields such as supply chain [8] and [9] or internet of things [10] and [11]. In order to fulfill this objective, ProKnow-C (Knowledge Development Process - Constructivist) methodology was applied [12].

The article is structured as follows. Section 2 presents the methodology adopted for the development of the research; followed by Section 3, which presents the processes adopted for the selection of articles. The analysis of the technical and qualitative aspects of the bibliometric portfolio, as well as the systemic portfolio analysis, are presented in sections 4 and 5, respectively. Finally, Section 5 brings the main conclusions.

\section{Methodological aspects}

In order to present the state of the art regarding the applicability of blockchain technology into the manufacturing industry, its current trends, existing gaps and to raise a related bibliographic portfolio, this study adopts a methodological procedure developed intended to present guidelines capable to guide a researcher towards the state of the art of a knowledge area. The methodology adopted is Knowledge Development ProcessConstructivist (hereinafter, ProKnow-C) developed by the Federal University of Santa Catarina [12].

The ProKnow-C methodology, as adopted, is presented in this study divided into three main steps: bibliographic portfolio selection, bibliographic portfolio analysis and systemic analysis. In the first step, the definition of the bibliographic portfolio, research axes are defined and broken down into keywords, which are the basis for the searches in the academic journal articles databases. Relevant works are filtered firstly by title reading followed by abstract reading. Pareto analysis was applied in order to find the most relevant works based on citations quantity as available in Google Scholar [13]. In the next step, the bibliographic portfolio analysis, journals' impact factor, keywords occurrence, year of publication, and most relevant authors are detailed. In the last step, the systemic analysis, the content of the works are analyzed, presenting the identified issues, the proposed deliveries and the gaps for further research.

Throughout the development of this work, software Mendeley was employed for bibliographic management and Microsoft Excel for data tabulation.

\section{Bibliographic portfolio selection}

The bibliographic portfolio selection consists of a raw base of articles collected from a set of keywords built under research axes.

As basis for the development of this investigation, two research axes were defined in order to synthesize the objectives of the study: (i) Blockchain; (ii) Industry. 
Subsequently, the defined axes were stratified into related keywords in order to cover a sufficiently representative amount of publications, as shown in Table 1.

Table 1. Search Axes and Keywords

\begin{tabular}{ll}
\hline Axes & Keywords \\
\hline Blockchain & Blockchain \\
\hline & Block-Chain \\
\hline & Block Chain \\
\hline Smart Contracts \\
\hline Crydustry & Engineering \\
\hline & Industry \\
\hline & Manufacturing \\
\hline & Cyber Physical Systems \\
\hline
\end{tabular}

In order to use such keywords for searches in databases using boolean operators, the defined keywords were grouped into the following search key: ("Blockchain" OR "Block-chain" OR "Block chain" OR "Smart contracts" OR "Cryptocontracts") AND ("Engineering” OR “Industry” OR “Manufacturing” OR "cyber physical systems")

Based on the alignment with the research interests, the following databases were selected: Scopus, ScienceDirect, Web of Science, ProQuest, Springer, and Ebsco. Access to the databases was facilitated through the CAPES (Coordination of Improvement of Higher Education Personnel) periodicals portal [14].

The filtering elements for the database consisted of limiting the results in scientific articles or conference papers published between 2014 and 2019. Searches into the databases were performed between June 6 and 14, 2019 and resulted in 1725 references, as shown in Table 2. As an initial activity in this step of filtering the base of papers, all duplicate references were removed, resulting in 1432 articles. This selection was managed using Mendeley, an open source reference management software.

Table 2. Database and Filtered Articles

\begin{tabular}{llll}
\hline Database & $\begin{array}{l}\text { Raw search } \\
\text { results }\end{array}$ & $\begin{array}{l}\text { Paper and conference } \\
\text { articles }\end{array}$ & Period: 2014-2019 \\
\hline Scopus & 1096 & 509 & 504 \\
\hline ScienceDirect & 59 & 51 & 50 \\
\hline Web of Science & 400 & 206 & 204 \\
\hline ProQuest & 3851 & 324 & 302 \\
\hline Springer & 706 & 525 & 502 \\
\hline Ebsco & 554 & 163 & 163 \\
\hline
\end{tabular}

Thereafter, each of the 1432 titles in the portfolio was read. Only 189 articles were selected due to their alignment with the research objective. As the next step, the abstracts 
were read and the papers that were not aligned were excluded, leaving 105 papers for the following analyzes.

Another filtering was performed aiming to identify the scientific recognition of the remaining articles. Through Google Scholar [13], the citations number for each article were verified on June 2019. Then, a Pareto analysis was performed to find the $20 \%$ of the articles that had approximately $80 \%$ of total citations. This first grouping of articles was characterized as repository $K$, and the remaining set was entitled as repository $P$, as shown in Figure 1.

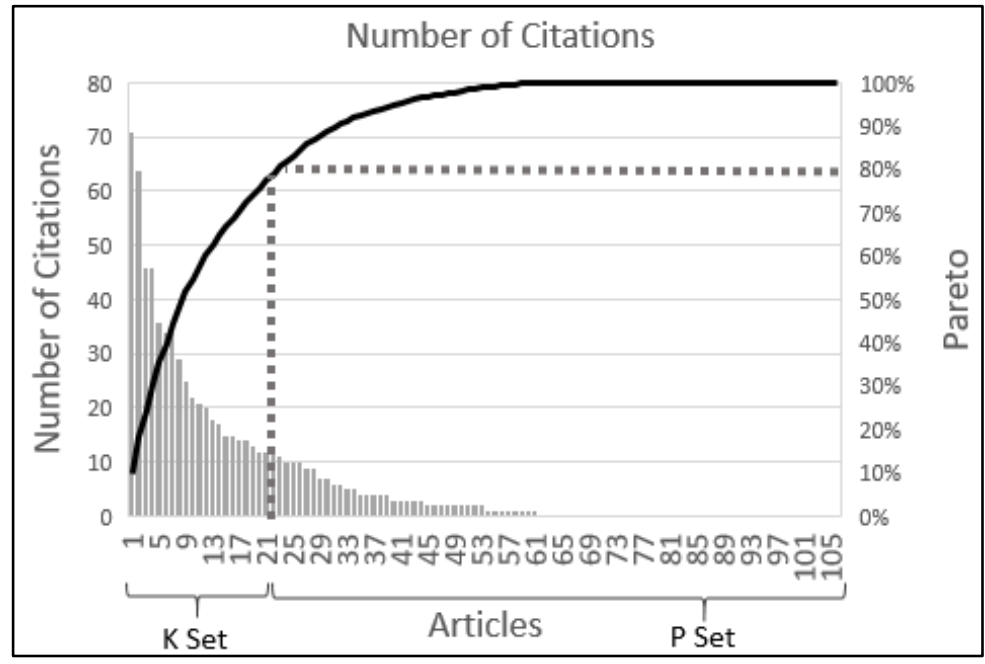

Figure 1. Number of citations per article and sets $\mathrm{K}$ and $\mathrm{P}$.

Initially, all articles in repository $K$ were pre-selected. A careful reading of the articles excluded two of them from the final portfolio, as they did not really meet the research focus.

From the P set, some articles were included in the selection as well, since despite gathering only a few citations, many of them were recent, and have not yet been deservedly appreciated by the scientific community. Therefore, some recent articles with few citations with relevant and diverse content were also included into the final set of selected articles, totaling 30 publications.

\section{Bibliometric analysis of bibliographic portfolio}

The bibliometric analysis allows evaluating and interpreting the bibliographic portfolio. Thus, it is possible to identify the relevance of journals and congresses about the research theme, the year of articles publication, the keywords found in the portfolio, and the authors relevance.

From the 30 selected publications, 22 are scientific papers from 18 journals. From these journals, a total of 13 have an impact factor on the JCR scale, 4 of which not being listed. Figure 2 shows the evaluated journals' impact factors.

The bibliometric portfolio presented the occurrence of 91 keywords. The most prominent keywords and their related occurrence are presented in Figure 2. From this list, keywords such as Blockchain, Industry 4.0 and Smart Contracts are represented by 
and related to the keywords also defined in the searches which resulted into the bibliographic portfolio selection. The remaining of the keywords are related directly to applications of the blockchain technology, in which supply chain and internet of things should be highlighted.

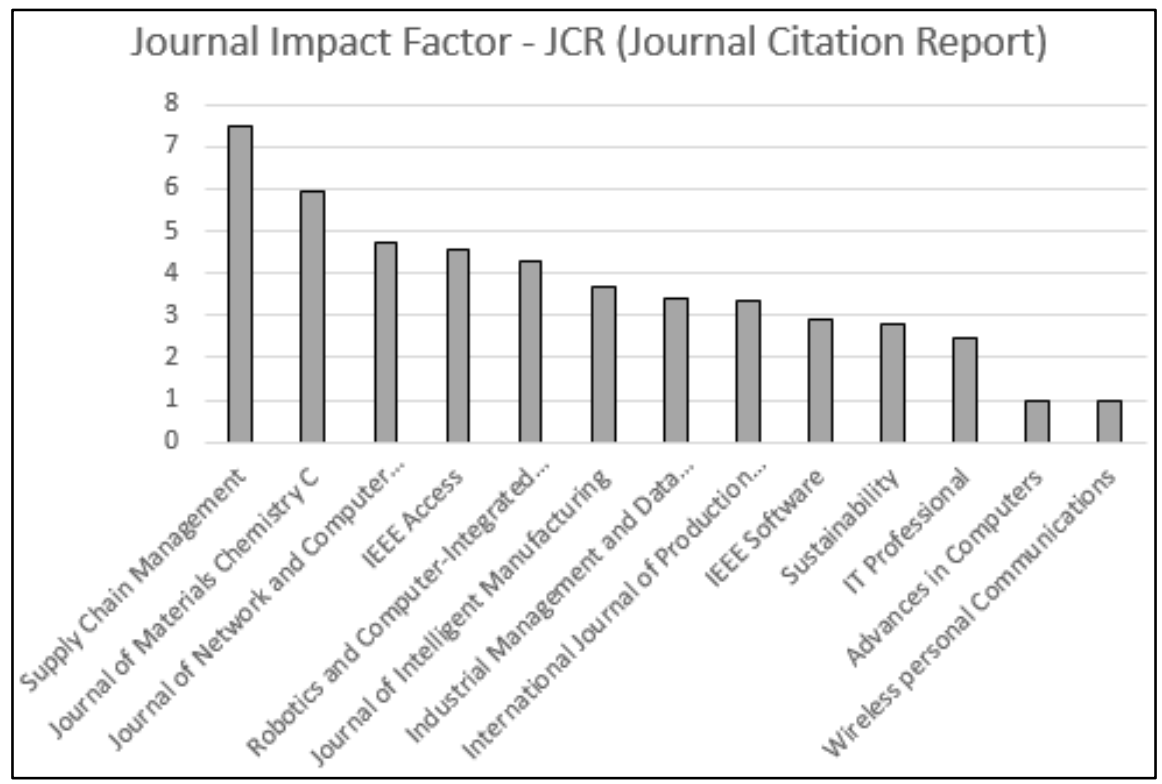

Figure 2. Journal Citation Report (JCR) Scale Impact Factor

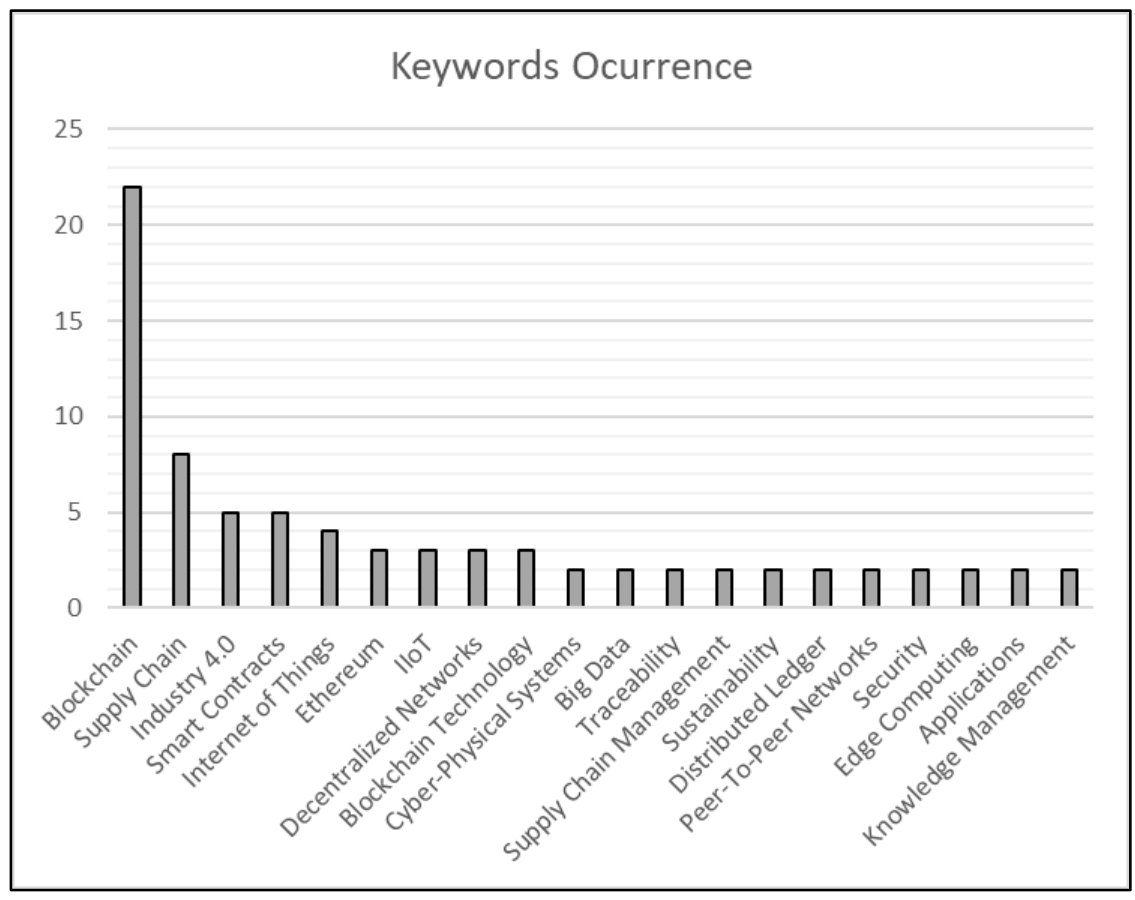

Figure 3. Occurrences of the most prominent keywords 
In order to observe the theme relevance evolution, data is presented in Table 3. It is noted that despite this research involved previous 5 years - from 2014 to 2019, relevant articles were selected only from 2017 on, showing the recent evolution of the scientific interest in this subject. In 2018 the number of publications increased considerably and in 2019 , considering that only the first semester publications were included, and also considering that they presented reduced citation opportunities - contributing to a possible non-selection in the bibliometric portfolio, it is expected a likely maintenance of the amount of publications.

Table 3. Number of Publications per year in the Bibliometric Portfolio

\begin{tabular}{ll}
\hline Year & Publication \\
\hline 2017 & 7 \\
\hline 2018 & 18 \\
\hline 2019 & 5 \\
\hline
\end{tabular}

A total of 85 authors composed the bibliographic portfolio, 79 of them presenting one work. From the 6 remaining authors, Mahtab Kouhizadeh, Joseph Sarkis, Wai Ming Wang, Layne Liu and Ali Vatankhah Barenji participated with 2 publications while Zhi Li presented 3 works.

\section{Systemic analysis}

The systemic analysis presents the content analysis of the bibliometric portfolio papers in order to summarize the presented issues, proposed deliverables, and identified research opportunities from critical analysis.

\subsection{Identified issues and proposed deliveries}

The studied publications can be categorized into five main groups according to the covered topics, as shown in Figure 4, namely: smart manufacturing, supply chain, security, knowledge sharing, and existing application studies.

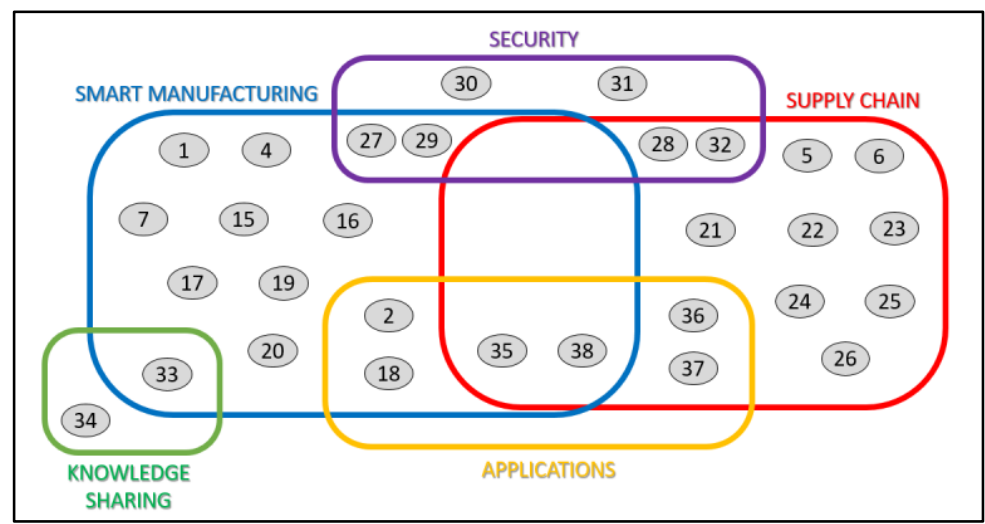

Figure 4. Categorization of bibliometric portfolio articles 


\subsubsection{Smart Manufacturing}

Articles under this category present strategies, methodologies, and frameworks for introducing blockchain technology directly into the industrial environment, with the main purpose of process optimization.

Two studies apply blockchain into disruptive technologies, such as [15] presenting a communication method between swarm systems, and [16] introducing the application of blockchain into a $5 \mathrm{G}$ network slicing methodology in an industrial environment in order to optimize traffic control on an internal network.

Two articles focus on machine-to-machine communication: [17] addresses the development of a decentralized manufacturing ecosystem, in which computer-controlled machines are directly connected to nodes of a blockchain network from which they can receive and respond to job requests. Similarly, [7] assesses the feasibility and considerations for applying smart contracts when developing such systems, presenting different blockchain architectures and comparing them to conventional automation systems.

Other authors develop solutions for industrial communities usage. [18] presents the concept of social manufacturing and how blockchain technology could be a transparent solution for trust in a socio-industrial environment. [4], in turn, applies blockchain technology for the registration of suppliers assessed based on their quality of service (QoS) for service composition. It assumes a network of cooperation between different companies in the same segment willing to share the QoS index of their suppliers.

Regarding communication and data management internally within a company, two studies remain. [19] presents a movement recording system based on accelerometers and extrapolation of results, seeking to list potential risks, quality prediction, trends, production monitoring, and fault diagnosis. Records are kept in blockchains, while sensitive data is kept off the network, only referred by the blockchain. Finally, [20] introduces an existing modular industrial middleware, Man4Ware, adding a previously unavailable blockchain functionality.

\subsubsection{Supply Chain}

In this category, the focus of the articles is limited to assessing the applicability of blockchain in supply chains to make them more efficient, transparent and secure.

Two papers developed smart contract product traceability frameworks, [5] and [6]. Exploring the feasibility and impacts of supply chain blockchain development are two studies. [21] presents the existing obstacles and barriers to the adoption of blockchain technologies in sustainable supply chain management. It concludes that, mostly, the problems are due to the immaturity of the technology. Paper from [22] brings together the applicability and impacts of using blockchain technology in four different theories of supply chain operation.

As specific solutions for supply networks, three works are presented. [23] proposes to use the Bitcoin blockchain as a solution for supply chain applications without the use of smart contracts. [24] develops a framework for controlling pollutant emission registration in the clothing industry. In [25], using smart contracts in the supply chain, in which both components of a product and the products themselves are taken as different tokens in a blockchain and the end product could be described as a recipe using component tokens. In this way, component tokens would be consumed when, in a supply network, a product was produced. Given this event, the product token would be created on the blockchain network and could then be transferred to the next consumer. 
Finally, [26] consolidates a standard methodology for developing supply chain blockchain solutions.

\subsubsection{Security}

Articles in this category provide secure and traceable solutions for industrial problems, whether falsification, property records or transparency.

Focused on developing tracking solutions are three works. [27] research is characterized as a supply chain article that proposes a product ownership registration system on the ethereum blockchain. It records the manufacturer, current owner, product shipments and receipts. By recording all transactions, both the ownership and originality of a product can be verified. [28] presents a framework for blockchain user authentication for applications in industry 4.0. Therefore, this article also falls into the smart manufacturing category. Also considered as an article in the Supply Chain category, work [29] suggests the use of records to control and monitor parts used in the assembly and maintenance of an aircraft, preventing counterfeiting.

Both [30] and [31] attempt to solve counterfeiting and copyright issues in additive manufacturing solutions using QR Codes and fluorescent signatures on material or RFID with serial numbers, both registered in a blockchain featuring a digital twin, representing the product virtually.

Two frameworks are proposed. Also categorized as a smart manufacturing article, [32] introduces a cloud manufacturing framework using blockchain to facilitate data transfer and service exchange in high security and eased traceability environment. [1] defines requirements for the creation of a "smart space" and how blockchain technology could be a solution to enable such a safely and reliably concept. The article was also characterized as smart manufacturing.

\subsubsection{Knowledge Sharing}

Both articles in this category present the possibility of using blockchain in knowledge management and sharing. Applying ontologies, edge computing, and blockchain, [33] presents a knowledge sharing system in an open manufacturing system. Therefore, it was also classified in this study as a smart manufacturing article. In turn, [34] presents a concept of knowledge management in the cloud. Two blockchains are presented, one protecting and providing encrypted knowledge, and the other keeping records and transactions of knowledge acquired by other blockchain participants.

\subsubsection{Applications}

Works under this topic gather different applications and specific uses of technology or studies regarding the possibility and feasibility of adopting such solutions. Also, mapping of general industry interest in these areas is also included. For example, [35] unites blockchain technology with IoT, presenting solutions for supply chain, autonomous vehicles and manufacturing asset management. Also presenting diversity in usage, [36] describes three application clusters related to logistics and supply chain. Additionaly, [37] focus on green supply chain solutions.

Aiming at Enterprise Resource Planning (ERP), [38] shows how blockchain technologies could be applied across different industry sectors, including a variety of use cases with application examples. Similarly, [2] critically reviews the benefits of using blockchain technologies and smart contracts in industry 4.0. It describes the necessary 
and sufficient conditions to be taken into consideration to define if a particular application requires the use of blockchain, seeking to restrain an improper use of technology just because of its strong disruptive tendency and hype.

\subsection{Identified gaps}

For the proposed problems and solutions, some gaps that could be filled were identified:

\subsubsection{Development}

Since not the full range of interesting solutions was developed for their applications, the following works presented several opportunities for additional features, functionalities, and developments.

[15] proposes the use of low power processors to reduce cost and increase autonomy, resulting in more advanced units in the swarm system.

Seeking to develop additional functionality for their systems, [23] and [27] suggest implementing incentive and reward systems for their blockchain miners. Similarly, [25] suggests the introduction of payment systems, loan control, and rents.

Two articles propose process automation. [30] presents the possibility of an automated anti-counterfeiting mechanism verification system - verification in the tested environment was manual. [17] recommends developing an automated system for reputation and trust verification in the decentralized manufacturing network.

\subsubsection{Implementation}

These works suggest the implementation of their concepts for verification of feasibility and applicability in different scenarios and scales for the proposed architectures validation.

Three works proposes to develop prototypes in order to test the feasibility of the proposals: [1] intents to verify the speed of the smart contract interoperability among smart spaces, [7] propose to check in a small scale the feasibility of the machine-tomachine communication system and [18] focuses on illustrating the proposed methods of credit assurance mechanism for manufacturing systems.

Intending to introduce the complete developed framework into practical situations, [4] suggests to integrate the proposed system into existing business process management solutions in order to automatize he evaluation of suppliers in service composition, [6] proposes to implement its system into business models that would benefit from open manufacturing focusing on physical traceability information sharing and [34] propose to apply its framework into different knowledge sharing scenarios.

Demanding additional analysis, [28] suggests to verify the applicabilty of the proposed system into smart factories and [32] proposes to evaluate the proposed architecture.

\subsubsection{Interfaces}

Both [1] and [17] recommend semantic and ontological development to enable communication and interoperability between machines or agents belonging to the industrial environment. 


\subsubsection{Security}

Publication [27] would seek to provide solutions for protecting the privacy of companies present in the blockchain and article [18] suggests investing in information security for social manufacturing resources.

\subsubsection{Further researches}

[21] and [22] raise several questions to be answered for a better understanding of the application of blockchain into supply chains, while [21] focuses on questions regarding the barriers presented for blockchain introduction in supply networks, [22] directs efforts into empirical generalized studies which could be applied to result in a better understanding and identification of the issues to be solved for the industrial applications.

[36] suggests exploring additional blockchain implementations made with related technical details, such as the identification of the node's responsible, consensus mechanisms applied and further details. Thus, it would be possible to observe the evolution of the technology implementation. Synergistically, [26] suggests extending the standard methodology of blockchain development to other applications, such as the automotive industry, focusing on vehicle identity and vehicle-to-vehicle communication and vehicle-infrastructure, towards autonomous driving and information exchange.

[16] proposes to evaluate the applicability of its study from the business model point of view, policies and legal perspectives, as well as other possible contexts.

Two works suggest studies concerning supply networks, [29] seeking to apply the concepts for spare parts supply and [37] presenting the need for a complete and detailed theoretical study of blockchain technologies evaluation applied to sustainable supply networks.

[31] proposes to consider products as smart products throughout the product life cycle, so that all failure and repair analysis can be used to improve component development, enabling product innovations development and introduction.

As challenges to be faced, [2] recommends exploring the following points: Scalability, low computing power blockchain application, definitions on which selection to adopt for consensus algorithms, privacy and security - data integrity, certifications, etc -, energy efficiency and latency, infrastructure issues, interoperability, standardization, legal and regulatory aspects. Similarly, [33] presents concerns regarding the security levels provided by the public blockchains concerning the amount of monitors, i.e. the exposure level of an agent.

\section{Conclusion}

The present work developed a study regarding the state of the art of the applicability of blockchain technology into industrial and manufacturing environments. Being explicit the direct relation of this subject with supply networks, and since this one presents strong relation and direct applicability of the technology, this tendency was evidenced. Besides, with the advent and eminence of developments in the so-called industry 4.0 and the urgency of applying different disruptive technologies in an industrial environment, intelligent manufacturing was found as another major core.

The main delivery and value of this research is related to the definition of research problems to be addressed, knowledge gaps to be closed and it is presented as a reference 
base of the current state of art of the applicability of the blockchain technologies in the manufacturing industry.

For the proper accomplishment of the developed research, the methodology ProKnow-C was applied, in order to raise bibliographic and systemic analyzes of the proposed theme, to identify trends and study opportunities. Thus, a portfolio of 30 articles was identified as representative for the given analysis, from an initial survey of 1432 publications presented between 2015 and 2019.

The ProKnow-C methology proved itself to be appropriate to the development of literature reviews. However, uncertanties and variation may be found. Its validity depends on a suitable selection of research axis and keywords definition for the adequate comprising of the research area. Different researchers may define different keywords resulting in distinct portfolios. Additionaly, the criteria in the definition of the filters may also imply in diverse results. Similar works may be developed with modifications into the keyword definition and filter criteria.

The gaps and opportunities identified mainly relate to the development of additional features to the originally proposed solutions, the implementation of these proposals in prototypes or controlled environments, the study of safety solutions for identified risks and a series of theoretical studies mainly concerning the applicability of the technology due to its still immature state of development.

In addition to the gaps previously identified, further research is suggested to identify solutions developed in other knowledge areas - such as food industry, pharmaceuticals, law, urbanism, among others - that could also prove useful for the manufacturing industry.

\section{References}

[1] N. Teslya and I. Ryabchikov. Blockchain-Based Platform Architecture For Industrial Iot. In: Conference Of Open Innovation Association, Fruct, Anais...Ieee, 2018.

[2] T.M. Fernandez-Carames and P. Fraga-Lamas. A Review On The Application Of Blockchain To The Next Generation Of Cybersecure Industry 4.0 Smart Factories. Ieee Access, vol. 7, 2019, pp. 4520145218.

[3] S. Nakamoto, 2008, Bitcoin: A Peer-To-Peer Electronic Cash System. Accesses: 15.07.2019. [Online]. Available: http://www.bitcoin.org.

[4] W. Viriyasitavat. Et Al. Blockchain-Based Business Process Management (Bpm) Framework For Service Composition In Industry 4.0. Journal Of Intelligent Manufacturing, 2018, pp. 1-12.

[5] Q. Lu and X. Xu. Adaptable Blockchain-Based Systems: A Case Study For Product Traceability. Ieee Software, vol. 34, 2017, pp. 21-27.

[6] H. Wu Et Al. A Distributed Ledger For Supply Chain Physical Distribution Visibility. Information (Switzerland), vol. 8, 2017, n. 4.

[7] M. Y. Afanasev Et Al. An Application Of Blockchain And Smart Contracts For Machine-To-Machine Communications In Cyber-Physical Production Systems. In: Proceedings - 2018 Ieee Industrial CyberPhysical Systems, Icps 2018, Anais...Ieee, 2018.

[8] Y. Wang. Et Al. Understanding Blockchain Technology For Future Supply Chains: A Systematic Literature Review And Research Agenda. Supply Chain Management. vol. 24 2019, pp. 62-84.

[9] M. Queiroz Et Al. Blockchain and supply chain management integration: a systematic review of the literature, Supply Chain Management: An International Journal. 2019.

[10] T.M. Fernandez-Carames and P. Fraga-Lamas. A Review on the Use of Blockchain for the Internet of Things Ieee Access, vol. 6, 2018, pp. 32979 - 33001.

[11] Q. Wang Et Al. Blockchain for the IoT and industrial IoT: A review. Internet of Things. 2019.

[12] L. Ensslin. Proknow-C, Knowledge Development Process-Constructivist. vol. 1, 2010.

[13] Google Schoolar, 2019. Accessed: 15.06.2019. [Online]. Available: https://scholar.google.com/

[14] Capes. Portal De Periódicos Da Capes., 2019. Accessed: 18.07.2019. [Online]. Available: http://www.periodicos.capes.gov.br. 
[15] E. C. Ferrer. The Blockchain: A New Framework For Robotic Swarm Systems. In: Advances In Intelligent Systems And Computing. vol. 881, 2019, pp. 1037-1058.

[16] J. Backman Et Al. Blockchain Network Slice Broker In 5G: Slice Leasing In Factory Of The Future Use Case. In: Joint 13Th Ctte And 10Th Cmi Conference On Internet Of Things - Business Models, Users, And Networks, Anais...Ieee, Nov. 2018.

[17] A. Angrish Et Al. A Case Study For Blockchain In Manufacturing: "Fabrec": A Prototype For Peer-ToPeer Network Of Manufacturing Nodes. Procedia Manufacturing, vol. 26, 2018, pp. 1180-1192.

[18] J.Liu, P. Jiang and J. Leng. A Framework Of Credit Assurance Mechanism For Manufacturing Services Under Social Manufacturing Context. In: Ieee International Conference On Automation Science And Engineering, Anais...Ieee, 2018.

[19] K. Chung Et Al. Blockchain Network Based Topic Mining Process For Cognitive Manufacturing. Wireless Personal Communications, vol. 105, 2019, pp. 583-597.

[20] N. Mohamed and J. Al-Jaroodi. Applying Blockchain In Industry 4.0 Applications. In: 2019 Ieee 9Th Annual Computing And Communication Workshop And Conference, Ccwc 2019, Anais...Ieee, 2019.

[21] S. Saberi Et Al. Blockchain Technology And Its Relationships To Sustainable Supply Chain Management. International Journal Of Production Research, vol. 57, 2019, pp. 2117-2135.

[22] H. Treiblmaier. The Impact Of The Blockchain On The Supply Chain: A Theory-Based Research Framework And A Call For Action. Supply Chain Management, vol. 23, 2018, pp. 545-559.

[23] M. Nakasumi. Information Sharing For Supply Chain Management Based On Block Chain Technology. In: 2017 Ieee 19Th Conference On Business Informatics (Cbi), Anais...Ieee, 2017.

[24] B. Fu, Z. Shu and X. Liu. Blockchain Enhanced Emission Trading Framework In Fashion Apparel Manufacturing Industry. Sustainability (Switzerland), vol. 10, 2018, n. 4.

[25] M. Westerkamp, F. Victor and A. Kupper. Blockchain-Based Supply Chain Traceability: Token Recipes Model Manufacturing Processes. Proceedings - Ieee 2018 International Congress On Cybermatics: 2018 Ieee Conferences On Internet Of Things, Green Computing And Communications, Cyber, Physical And Social Computing, Smart Data, Blockchain, Computer And Information Technology, 2018, pp. 15951602 .

[26] G. Perboli, S. Musso and M. Rosano. Blockchain In Logistics And Supply Chain: A Lean Approach For Designing Real-World Use Cases. Ieee Access, vol. 6, 2018, pp. 62018-62028.

[27] K. Toyoda Et Al. A Novel Blockchain-Based Product Ownership Management System (Poms) For AntiCounterfeits In The Post Supply Chain. Ieee Access, vol. 5, 2017, pp. 17465-17477.

[28] C. Lin Et Al. Bsein: A Blockchain-Based Secure Mutual Authentication With Fine-Grained Access Control System For Industry 4.0. Journal Of Network And Computer Applications, vol. 116, 2018, pp. 42-52.

[29] Y. Madhwal and P. Panfilov. Blockchain And Supply Chain Management: Aircrafts' Parts' Business Case. Annals Of Daaam \& Proceedings, vol. 28, 2017, pp. 1051-1056.

[30] Z. C. Kennedy Et Al. Enhanced Anti-Counterfeiting Measures For Additive Manufacturing: Coupling Lanthanide Nanomaterial Chemical Signatures With Blockchain Technology. Journal Of Materials Chemistry C, vol 5, 2017, pp. 9570-9578.

[31] M. Holland, C. Nigischer and J. Stjepandic. Copyright Protection In Additive Manufacturing With Blockchain Approach. (Chen, Ch And Trappey, Ac And Peruzzini, M And Stjepandic, J And Wognum, N, Ed.) In: Advances In Transdisciplinary Engineering, Anais... vol.5, 2017, pp 914-921.

[32] Z. Li, A. V. Barenji and G. Q. Huang. Toward A Blockchain Cloud Manufacturing System As A Peer To Peer Distributed Network Platform. Robotics And Computer-Integrated Manufacturing, vol. 54, 2018, pp. 133-144.

[33] Z, Li Et Al. Toward Open Manufacturing A Cross-Enterprises Knowledge And Services Exchange Framework Based On Blockchain And Edge Computing. Industrial Management And Data Systems, vol. 118, 2018, pp. 303-320.

[34] Z. Li Et Al. Cloud-Based Manufacturing Blockchain: Secure Knowledge Sharing For Injection Mould Redesign. Procedia Cirp, vol. 72, 2018B, pp. 961-966.

[35] D. Miller. Blockchain And The Internet Of Things In The Industrial Sector. It Professional, vol. 20, 2018, pp. 15-18.

[36] M. Petersen, N. Hackius and B. Von See. Mapping The Sea Of Opportunities: Blockchain In Supply Chain And Logistics. It - Information Technology, vol. 60, 2018, pp. 263-271.

[37] M. Kouhizadeh and J. Sarkis. Blockchain Practices, Potentials, And Perspectives In Greening Supply Chains. Sustainability (Switzerland), vol. 10, 2018, pp. 3652.

[38] A. Banerjee. Blockchain Technology: Supply Chain Insights From Erp. In: Raj, P.; Deka, G. C. B. T.-A. In C. (Ed.). Advances In Computers. Advances In Computers. [S.L.] Elsevier, 2018, pp. 69-98. 